\title{
Traduire
}

Revue française de la traduction

Traducteurs en blouse blanche

\section{Pluriel caché dans les noms composés en anglais médical}

Serge Quérin

\section{OpenEdition}

1 Journals

\section{Édition électronique}

URL : http://journals.openedition.org/traduire/909

DOI : $10.4000 /$ traduire.909

ISSN : 2272-9992

\section{Éditeur}

Société française des traducteurs

\section{Édition imprimée}

Date de publication : 1 juin 2017

Pagination : 13-19

ISSN : 0395-773X

\section{Référence électronique}

Serge Quérin, «Pluriel caché dans les noms composés en anglais médical », Traduire [En ligne],

236 | 2017, mis en ligne le 01 juin 2019, consulté le 30 juin 2019. URL : http://journals.openedition.org/ traduire/909 ; DOI : 10.4000/traduire.909 


\section{Pluriel caché \\ dans les noms composés \\ en anglais médical}

\section{Serge Quérin}

On sait que l'adjectif est invariable en anglais et qu'il en va de même du premier élément d'un nom composé 'nom + nom', qui fait alors fonction d'adjectif. La traduction de ces noms composés à pluriel caché ne pose généralement aucun problème dans la langue courante, a flower garden devenant tout naturellement un jardin de fleurs et a stamp collection, une collection de timbres. Le problème ne se pose tout simplement pas dans le cas de figure où le premier élément porte d'emblée la marque du pluriel en anglais, le singulier n'étant pas envisagé : a clothes brush devient une brosse à habits et a goods train, un train de marchandises.

Il en va autrement dans les vocabulaires spécialisés. Le domaine de l'éducation nous en fournit un bon exemple. En formation professionnelle, notamment dans les domaines de la santé, on a de plus en plus recours à l'approche par compétences, ou competency-based approach en anglais. Le premier élément du terme anglais est remarquable à la fois par le pluriel qui s'y cache et par le paronyme avec lequel il peut être confondu. II faut en effet distinguer la compétence (competence) et les compétences (competencies). Une compétence (a competency) est une aptitude observable qui se développe par étapes, du clinicien débutant au clinicien expert. En revanche, la compétence (competence) est l'ensemble des aptitudes démontrées dans de multiples domaines ou aspects du rendement; elle est limitée par le contexte de pratique de chacun et évolue dans le temps (Collège royal des médecins et chirurgiens du Canada, 2016). Or, bien que la compétence soit le but ultime de toute formation professionnelle, l'approche par compétences consiste bel et bien à faire acquérir à l'apprenant diverses compétences, que l'on peut observer et évaluer à des étapes successives de sa formation.

Dans le domaine médical, de très nombreux noms composés en anglais dont le premier élément cache un pluriel sont susceptibles d'être trop rapidement rendus entièrement au singulier en français, que ce soit à l'écrit par le traducteur ou à l'oral comme à l'écrit par le médecin, ce dernier étant pourtant bien au fait de la notion désignée par le terme en question. Ce type d'erreur, l'une des nombreuses difficultés rencontrées en traduction médicale (Quérin, 2017), 
peut avoir plusieurs explications : pluriel qui s'impose logiquement en français mais qui peut échapper à la traduction par méconnaissance du signifié ; singulier concevable, mais primauté du pluriel sur le plan idiomatique en français, pouvant ne pas être prise en compte par le traducteur ou le locuteur ; voire risque de confondre le premier élément ou une partie de celui-ci avec un véritable adjectif en français. La prédominance de la langue anglaise dans la transmission du savoir médical et les correspondances variables entre disease et sickness d'une part et maladie et mal d'autre part (Audet et Vandaele, 2003) forment la toile de fond sur laquelle ces erreurs de traduction tendent à se produire et à se perpétuer.

Les listes qui suivent ont été établies en parcourant les entrées asthma, disease, lung, pneumoconiosis, sickness, study et syndrome des dictionnaires médicaux bilingues Gladstone (2002) et Dorland (Maniez, 2009), et toutes celles du Vocabulaire panlatin des pneumopathies professionnelles publié par l'Office québécois de la langue française (2012) en collaboration avec le réseau Realiter. Quelques termes ont été exclus du relevé après avoir vérifié la fréquence relative des diverses variantes possibles au moyen du moteur de recherche Google et constaté que, tout compte fait, le pluriel était plus souvent marqué que caché en anglais. Nous avons également consulté la base de données LiSSa et le MeSH bilingue anglais-français afin d'identifier ou confirmer les diverses variantes possibles du terme correspondant en français. Enfin, une expérience clinique d'une trentaine d'années au Québec et des contacts fréquents avec la France nous ont permis de repérer, à l'oral ou à l'écrit, des formes fautives (ou, du moins, non idiomatiques) et des variantes régionales couramment employées.

Pluriel qui s'impose logiquement en français mais qui peut échapper à la traduction par méconnaissance du signifié

amniotic band syndrome $\rightarrow$ maladie(1) des brides amniotiques

brittle bone disease $\rightarrow$ maladie des os de verre(2)

case control study $\rightarrow$ étude cas-témoins

cat-scratch disease $\rightarrow$ maladie des griffes du chat

cold agglutinin disease $\rightarrow$ maladie des agglutinines froides

cytomegalic inclusion disease $\rightarrow$ maladie des inclusions cytomégaliques

dense deposit disease $\rightarrow$ maladie(3) à dépôts denses

hand, foot and mouth disease $\rightarrow$ syndrome (4) mains-pieds-bouche

heavy chain disease $\rightarrow$ maladie des chaînes lourdes

hyaline membrane disease $\rightarrow$ maladie des membranes hyalines

immune complex disease $\rightarrow$ maladie à complexes immuns

(1) Les termes syndrome et maladie sont parfois interchangeables, comme c'est le cas ici.

(2) Mieux connue sous son nom scientifique d'ostéogenèse imparfaite.

(3) Ou glomérulonéphrite.

(4) Ou maladie. 
inclusion cell disease $\rightarrow$ maladie des cellules à inclusions

lazy leukocyte syndrome $\rightarrow$ syndrome des leucocytes paresseux

light chain disease $\rightarrow$ maladie des chaînes légères

polycystic ovary syndrome $\rightarrow$ syndrome des ovaires polykystiques

resistant ovary syndrome $\rightarrow$ syndrome des ovaires résistant(s) aux gonatrophines(5)

restless leg syndrome(6) $\rightarrow$ syndrome des jambes sans repos

sleep apnea syndrome $\rightarrow$ syndrome d'apnées du sommeil

white spot disease $\rightarrow$ morphée en gouttes

yellow nail syndrome $\rightarrow$ syndrome des ongles jaunes

Singulier concevable, mais primauté du pluriel sur le plan idiomatique en français, pouvant ne pas être prise en compte par le traducteur ou le locuteur

bird-breeder's disease(7) $\rightarrow$ maladie des éleveurs d'oiseaux (8)

caisson disease $(9) \rightarrow$ maladie des caissons $(10)$

cheese washer's lung(11) $\rightarrow$ maladie des laveurs de fromage(12)

compartment syndrome $\rightarrow$ syndrome des loges(13)

detergent worker's disease $\rightarrow$ maladie des ouvriers des usines de détergents

fish meal worker's lung $\rightarrow$ poumon des travailleurs des abats de poissons

flax dresser's disease $\rightarrow$ maladie des travailleurs du lin

furrier's lung $\rightarrow$ poumon des fourreurs

hard-metal pneumoconiosis $\rightarrow$ maladie des métaux durs(14)

(5) Ou aux gonadostimulines. Ce syndrome est mieux connu sous le nom d'insuffisance ovarienne primitive.

(6) On rencontre aussi la forme restless legs syndrome, dans laquelle le pluriel est marqué.

(7) Le pluriel est parfois marqué en anglais (bird-breeders' disease). On rencontre également des variantes avec pigeon- (maladie des éleveurs de pigeons) et avec fancier's (ou fanciers') au lieu de breeder's (ou breeders'), de même sens.

(8) La présente liste comprend de nombreuses appellations de pneumopathies professionnelles et dans chacune d'elles les mots disease et maladie sont parfois remplacés respectivement par lung et poumon (et vice-versa). On peut, par exemple dans le cas présent, rencontrer bird-breeder's lung $\rightarrow$ poumon des éleveurs d'oiseaux. Certaines variantes sont construites autour des termes asthma (asthme) et cough (toux).

(9) Mieux connue sous le nom de decompression sickness et parfois encore appelée caisson sickness.

(10) Aussi connue sous les noms de mal de décompression, maladie des plongeurs et maladie des scaphandriers.

(11) Aussi connu sous le nom de cheese handler's disease.

(12) Aussi connue sous le nom de poumon de fromager.

(13) Le terme syndrome du compartiment est employé au Québec (variation terminologique). Le singulier, ici, témoigne du fait qu'un seul compartiment (ou loge) musculaire est habituellement atteint chez un patient donné, alors que syndrome des loges renvoie aux différentes loges pouvant être atteintes d'un sujet à un autre en fonction du siège de la lésion musculaire initiale.

(14) Aussi connue sous les noms de pneumoconiose par métaux durs et pneumoconiose à métaux durs. 
hairspray disease $\rightarrow$ maladie des coiffeurs

humidifier lung (15) $\rightarrow$ maladie des humidificateurs

legionnaire's disease (16) $\rightarrow$ maladie des légionnaires $(17)$

maple syrup urine disease $\rightarrow$ maladie des urines à odeur de sirop d'érable

milk drinker's syndrome $\rightarrow$ syndrome des buveurs de lait

mixed dust pneumoconiosis $\rightarrow$ pneumoconiose à poussières mixtes(18)

motion sickness $\rightarrow$ mal des transports

mountain sickness $\rightarrow$ mal des montagnes

mushroom worker's disease (19) $\rightarrow$ maladie des champignonnistes

pituitary snuff taker's lung $\rightarrow$ pneumonite d'hypersensibilité aux extraits hypophysaires prion diseases $\rightarrow$ maladies à prions (20)

radiation sickness $\rightarrow$ maladie des rayons

silver polisher's lung disease $\rightarrow$ maladie des polisseurs d'argenterie

slate worker's lung $\rightarrow$ maladie des ardoisiers(21)

staphylococcal scalded skin syndrome(22) $\rightarrow$ syndrome des enfants ébouillantés

tanner's disease $\rightarrow$ rossignol(23) des tanneurs(24)

wheat weevil disease $\rightarrow$ maladie des charançons de blé

woodworker's lung $\rightarrow$ maladie des travailleurs du bois

vineyard sprayer's disease $\rightarrow$ maladie des vignerons

Risque de confondre le premier élément ou une partie de celui-ci avec un véritable adjectif en français

antiphospholipid syndrome $\rightarrow$ syndrome des antiphospholipides (et non syndrome *antiphospholipide)

neuroleptic malignant syndrome $\rightarrow$ syndrome malin des neuroleptiques (et non syndrome *neuroleptique malin)

(15) Parfois appelé air conditioner lung.

(16) On rencontre aussi la forme legionnaires' disease, dans laquelle le pluriel est marqué.

(17) Aussi appelée légionellose, cette maladie infectieuse a fait son apparition lors d'un congrès de l'American Legion à Philadelphie en 1976. La forme maladie du légionnaire est moins courante mais correcte.

(18) Aussi connue sous le nom de pneumoconiose mixte.

(19) Aussi connue sous le nom de mushroom picker's disease. On rencontre parfois le pluriel : mushroom workers' disease ou mushroom pickers' disease.

(20) On rencontre aussi la forme maladie à prion.

(21) Aussi appelée schistose.

(22) À distinguer du scalded skin syndrome (tout court) qui est l'érythrodermie bulleuse avec épidermolyse.

(23) Nom donné à cause des cris de douleur que la souffrance provoque (Robert), les lésions aux mains résultant de l'action caustique du chrome.

(24) Affection aussi appelée pigeonneau, par comparaison avec les pattes de l'oiseau (Robert). 


\section{Quand doit-on suspecter ces pièges de traduction, et comment les éviter?}

En parcourant les listes qui précèdent, on constate qu'en présence d'un nom composé en anglais médical à deux ou trois éléments ('nom + nom', 'nom + nom + nom' ou 'adjectif + nom + nom'), le ou les premiers éléments font fonction d'adjectif et peuvent receler un pluriel caché à prendre en compte dans la traduction française. Ce pluriel peut passer inaperçu surtout par méconnaissance de la notion désignée (le traducteur supposant, par exemple, que le polycystic ovary syndrome n'affecte qu'un ovaire) ou parce qu'on ignore qu'à quelques variations régionales près (résultant d'une plus grande influence de l'anglais, par exemple), le pluriel est plus idiomatique en français, même si le singulier ne heurte pas la logique. Ces noms composés à substantif(s) adjectivé(s) sont habituellement traduits par une locution nominale nom + préposition + nom, construite le plus souvent autour de de, habituellement contracté avec l'article défini pluriel (d'où des), ou encore élidé (d'), et plus rarement à l'aide de à, voire en. Il est beaucoup plus rare (nous n'en avons relevé que deux exemples) que la méprise soit totale et que le substantif adjectivé en anglais soit rendu à tort par un adjectif en français. C'est en effet forcer un peu l'hypallage que d'évoquer, par exemple, un syndrome *antiphospholipide quand ce sont en fait les anticorps à l'origine du syndrome qui sont antiphospholipides, d'où l'appellation correcte de syndrome des antiphospholipides.

II faut donc toujours être prudent devant un nom composé en anglais, en particulier quand il désigne une maladie professionnelle, dont on a vu de nombreux exemples dans les listes qui précèdent, en grande majorité des pneumopathies. La préférence du français pour les formes au pluriel est habituelle pour les maladies professionnelles, toutefois elle n'est pas constante : pour preuve l'existence d'un poumon de fermier (farmer's lung) et d'un poumon de fromager, ce dernier terme étant en concurrence avec maladie des laveurs de fromage, comme on l'a vu. La prudence est en fait de mise devant tout terme désignant une maladie (disease), un mal (sickness) ou un syndrome. Au moment de traduire brittle bone disease, par exemple, il faut se demander s'il est plausible que cette maladie n'affecte qu'un os, ou encore qu'en la nommant en français on ait donné à os au singulier un sens collectif (" tous les os "), en optant pour maladie *de l'os de verre. On se rendra sans doute compte, même en rencontrant le terme anglais pour la première fois, qu'il est beaucoup plus naturel de le rendre en français par maladie des os de verre.

Il faut enfin entrevoir dans certains cas une éventuelle modulation (par exemple, disease $\rightarrow$ syndrome, hairspray disease $\rightarrow$ maladie des coiffeurs, mais aussi white spot disease $\rightarrow$ morphée en gouttes) et qu'il puisse exister diverses variantes admises, régionales ou non, dont les notes de bas de page dans cet article ont donné de nombreux exemples.

Tout compte fait, la meilleure stratégie de traduction est d'abord de s'assurer de bien maîtriser la notion sous-jacente, puis de consulter de multiples sources, comme nous l'avons fait pour 
établir les listes proposées ici : dictionnaires médicaux bilingues, vocabulaire ou lexique spécialisé, base de données en ligne et même ce bon vieux Google, fort utile pour le problème qui nous occupe, qui a trait à des entités souvent relativement rares (bien peu de cliniciens ayant vu un cas de maladie des ardoisiers, par exemple, et encore moins publié sur le sujet). Ce moteur de recherche généraliste ratisse bien sûr dans tous les registres, mais il a le mérite de refléter l'usage par un bien plus grand nombre d'occurrences que ce que des bases de données plus spécialisées comme PubMed peuvent consigner. À chaque problème, sa solution.

serge.querin@umontreal.ca

Serge Quérin est médecin et professeur à la Faculté de médecine de l'Université de Montréal. Passionné de terminologie et de traduction médicale, il est l'auteur du Dictionnaire des difficultés du français médical, dont la 3e édition est parue en 2017. II a également rédigé des articles portant sur la variation terminologique en médecine, notamment sur les termes hybrides grécolatins et les québécismes médicaux. Sous le pseudonyme @terminomed, il anime depuis 2014 un fil Twitter consacré à la terminologie médicale.

\section{Bibliographie}

AUDET Geneviève et VANDAELE Sylvie, 2003, Disease, illness, sickness et pathology : faut-il en faire une maladie ? Kirkland, Groupe traduction - Les compagnies de recherche pharmaceutique du Canada, http://www.groupetraduction.ca/documents/Vol14no42003.pdf, consulté le 4 mars 2017.

Catalogue et index des sites médicaux en langue française, LiSSa - Littérature scientifique en santé, France, CHU de Rouen - CiSMeF, http://www.lissa.fr, consulté le 04/03/2017.

Collège royal des médecins et chirurgiens du Canada, 2016, La compétence par conception (CPC). Foire aux questions. Partie 3 : Termes et usages. Collège royal des médecins et chirurgiens du Canada,

http://www.royalcollege.ca/rcsite/documents/cbd/cbd-handout-glossary-april-27-f.pdf, consulté le 10/01/2017.

GLADSTONE William J., 2002, Dictionnaire anglais-français de sciences médicales et paramédicales, 5e édition, Saint-Hyacinthe (Canada) et Paris, Edisem et Maloine.

Institut national de la santé et de la recherche médicale, MeSH bilingue anglais-français, France, Institut national de la santé et de la recherche médicale, http://mesh.inserm.fr, consulté le 04/03/2017. 
Le Grand Robert de la langue française, version numérique, 2013, Paris, Dictionnaires Le Robert. MANIEZ François (coord. de l'édition française), Dorland - Dictionnaire médical bilingue, 2009, Issy-les-Moulineaux (France), Elsevier Masson.

Office québécois de la langue française, 2012, Vocabulaire panlatin des pneumopathies professionnelles, Québec (Canada), Office québécois de la langue française, https://www.oglf.gouv.qc.ca/ressources/bibliotheque/dictionnaires/panlatin_pneumopathies 20130124.pdf, consulté le 04/03/2017.

QUÉRIN Serge, 2017, Dictionnaire des difficultés du français médical, 3e édition, Saint-Hyacinthe (Canada) et Paris (France), Edisem et Maloine. 